\title{
Light Sheet Microscopy with LED illumination
}

Gianmaria Calisesi, Michele Castriotta, Andrea Farina, Cosimo

D'Andrea, Gianluca Valentini, Andrea Bassi

Politecnico di Milano, Italy

\section{Abstract Text}

Light emitting Diodes (LED) are usually not considered a good light source for Light Sheet Fluorescence Microscopy because the lack of spatial coherence makes it difficult to tightly focus light along one direction. However, using LED in fluorescence microscopy brings advantages such as color availability, low cost, and reduced presence of speckle patterns. In Light Sheet Fluorescence microscopy this can avoid the typical shadowing artifacts. We show that, upon selectively illuminating a volume of the sample with LEDs, we can reconstruct it in three dimensions. The volumetric light shaping is possible thanks to a spatial light modulator, which is coupled to an illumination orthogonal to the detection axis. In this configuration, said modulation happens within the depth of field of the detection objective ( $\Delta \mathrm{z}$ in Fig.1). After the acquisition of $\mathrm{N}$ patterns, an inversion problem is solved, resulting in the sample volumetric reconstruction. This modulation approach can only reconstruct a thin volume of the sample, according to the illumination optics depth of focus [1]. In order to extend the reconstructed volume, we scan the illumination patterns along the illumination axis, by means of a fast translating stage. The synchronization of the motion with the acquisition of a CMOS camera, enables a reconstruction volume of $3 \mathrm{~mm} \times 3 \mathrm{~mm} \times 0.1 \mathrm{~mm}$ with isotropic resolution $(2.5 \mu \mathrm{m})$. The reconstructions resolution is limited by the finest degree with which is possible to spatially modulate light, ultimately the spatial modulator pixel size.

Furthermore, we propose the use of patterned acquisition for Compressive Sensing, a signal analysis technique that reduces the number of modulation patterns to be acquired for a 3-dimensional reconstruction. This is achieved upon solving an ill-posed problem 
with further mathematical constraints which are related to the sample spatial features. The technique yields an accurate reconstruction of the sample anatomy even at significant compression ratios, achieving higher volumetric acquisition rate and eventually reducing photodamage on biological samples.

We demonstrate the absence of shadowing artifacts and compression capability of the technique by imaging chemically cleared fluorescent mouse and adult zebrafish brains and zebrafish embryos in vivo.

$a$

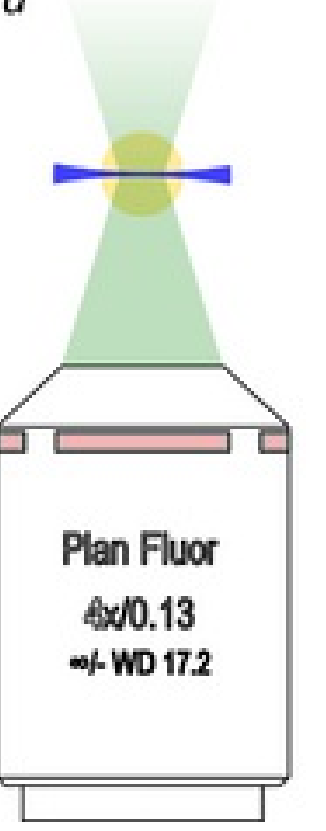

$b$

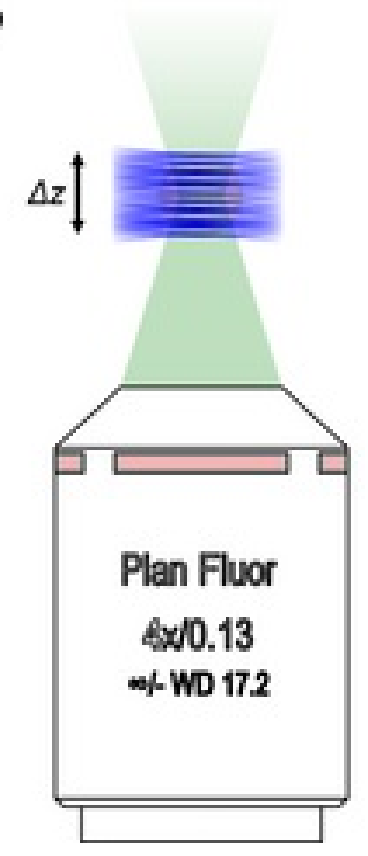

Figure 1: The illumination is confined to a single plane in LSFM (a). Selected Volume Illumination in the presented setup: the entire depth of field of the detection objective is illuminated and axially modulated (b) 